\title{
Public health staff will have pensions invested in tobacco under transfer to local government schemes
}

Clarification-In this News article by Jonathan Gornall (BMJ 2013;346:f680, doi:10.1136/bmj.f680), the author suggests the following clarification of the NHS pensions situation:

"Continued access to the NHS Pension Scheme may also be allowed for those who already have access to the NHSPS and who are recruited as 'new joiners' to public health roles when they are 'nearing retirement.' In such cases it is being left to local authorities to decide 'whether to apply for a directions order."”

Cite this as: BMJ 2013;346:f1007

๑ BMJ Publishing Group Ltd 2013 